\title{
Education à l'énergie : pratiques enseignantes et représentations des élèves
}

\author{
Energy Education: Teaching Practices and Student
}

Representations

Hugon Mandarine ${ }^{1}$, Maguin Françoise ${ }^{2}$, Bois Evelyne ${ }^{3}$, Glomeron Frédéric $^{4}$

${ }^{1}$ ERCAE, Université d'Orléans, ESPE CVL, mandarine.hugon@univ-orleans.fr

${ }^{2}$ ERCAE, Université d'Orléans, francoise.maguin@univ-orleans.fr

${ }^{3}$ ERCAE, Université d'Orléans, ESPE CVL, evelyne.bois@univ-orleans.fr

${ }^{4}$ ERCAE, Université d'Orléans, frederic.glomeron@univ-orleans.fr

RÉSUMÉ. Dans la perspective d'une transition énergétique, cette recherche questionne les pratiques enseignantes d'éducation aux économies d'énergie en collège : ces pratiques ont-elles un effet sur les représentations et pratiques des élèves-adolescents ? D'après l'analyse de cinq entretiens réalisés auprès d'enseignants d'un même collège, peu de gestes concrets réfléchis collectivement et réalisés librement sont proposés aux élèves, même si nous repérons une nuance en fonction des disciplines enseignées. Néanmoins, l'analyse de 257 questionnaires distribués auprès d'élèves de Troisième montre que la plupart des adolescents interrogés connaissent les éco-gestes à mettre en œuvre au quotidien et leurs effets. Mais la pratique de ces éco-gestes semble liée au milieu de vie sociale, aux pratiques familiale et scolaire. Notre étude nous amène alors à interroger la co-construction des actions éducatives entre une diversité d'acteurs, ce notamment dans la perspective de l'évolution des programmes scolaires.

ABSTRACT. With the prospect of an energy transition, this study questions teaching practices on energy education in French middle school: do these practices have an effect on the representations and practices of student-teenagers? Based on the analysis of five interviews with teachers in the same middle school, there are few concrete, collectively thought-out and freely realized actions that are offered to students, even if we identify a nuance according to the disciplines taught. Nevertheless, the analysis of 257 questionnaires distributed to French middle school students shows that students generally seemed aware of energy saving actions' effects and of their implementing. However, their actions seem to be influenced by academic knowledge, family practices and social environment. Our study then leads us to question the co-construction of educational actions between a variety of actors, particularly in view of the evolution of school curricula.

MOTS-CLÉS. Adolescents, Collège, Education à l'énergie, Pratiques enseignantes, Représentations sociales.

KEYWORDS. Teenagers, Middle School, Energy education, Teaching practices, Social representations.

\section{Transition énergétique et enseignement des éco-gestes}

Dans la perspective de la transition énergétique (Loi du 17 août 2015) visant des transformations de la production, de la distribution et de la consommation d'énergie, les textes institutionnels incitent à former et éduquer les citoyens de demain aux économies d'énergie inscrites dans une démarche de développement durable. Or, pour évoluer vers ce changement, cela nécessite de questionner les habitudes comportementales et de développer chez les élèves des dispositions à choisir, décider et s'engager (Lange, 2008), ce qui correspond à «éduquer au choix et non $[. .$.$] enseigner des choix » 1$. Le poids des habitudes

\footnotetext{
${ }^{1}$ Circulaire $N^{\circ}$ 2007-077 du 29-3-2007, Seconde phase de généralisation de l'éducation au développement durable (EDD).
} 
et le rapport à la technique peuvent effectivement être un frein au changement, « moment de la dynamique sociale », produisant un bouleversement des systèmes de valeur, des univers cognitifs, etc. (Zelem, 2010). Il faut également s'assurer que les élèves maîtrisent suffisamment de savoirs afin d'avoir plus «de probabilité pour que [leurs] attitudes sociales s'inspirent des savoirs » (Audigier, 2007, p. 31). Le rôle de l'enseignant doit alors permettre aux élèves d'agir à l'école et de transférer les savoirs et actions chez eux et dans leur communauté (Legardez, 2004). Ces modalités d'enseignement rejoignent celles que les enseignants sont désormais invités à développer de façon plus générale dans le cadre de l'éducation à la citoyenneté (développer l'esprit critique, éduquer aux comportements responsables, favoriser la participation et l'engagement des élèves, etc.). Cependant, en ce qui concerne l'Education au Développement Durable (EDD), les enseignants se sentent souvent démunis, sortent difficilement du cadre scolaire et disciplinaire, ce qui ne favoriserait pas le changement souhaité et la transition vers de nouveaux comportements. Dans la présente étude, nous nous centrons plus précisément sur les pratiques d'éducation à l'énergie : les enseignants rencontrent-ils les mêmes obstacles?

Les économies d'énergie renvoient à de multiples activités (Bonfils, 2008 ; GarabuauMoussaoui, 2009, 2011 ; Zelem 2010) pour limiter la consommation d'énergie. Il s'agit d'éviter les pertes portant sur l'énergie par un engagement de l'individu dans une société de «modération» (Moussaoui, 2007) sur la base du juste nécessaire, et non uniquement en suivant un principe de "consommation ». La démarche adoptée serait celle d'un citoyen éco-responsable. Les enseignants de collège encouragent-ils les élèves à développer cette démarche?

Dans cette étude, nous interrogeons, dans un premier temps, les représentations sociales et l'engagement des enseignants vis-à-vis des économies d'énergie ainsi que leurs pratiques pédagogiques. La définition du développement durable étant complexe et liée à la façon dont «nous percevons notre relation au monde » (Jickling, 1998, cité par Barthes et Jeziorski, 2012, p. 65), étudier les représentations sociales ${ }^{2}$ (Jodelet, 1989 ; Seca, 2010) chez les enseignants est en effet nécessaire pour comprendre leurs pratiques. De nombreuses études ayant montré des représentations sociales variables selon le contexte et « un décalage entre la définition institutionnelle et les savoirs sociaux du développement durable » (Barthes et Jeziorski, 2012, p. 66), il nous parait alors important de questionner à la fois les représentations sociales et l'engagement des enseignants vis-à-vis des économies d'énergie pour faire le lien avec leurs pratiques pédagogiques et ce, au sein d'un même établissement pour limiter l'effet de contexte.

Dans un second temps, nous nous interrogeons quant à la mise en acte des éco-gestes par les collégiens eux-mêmes. Connaissent-ils les gestes à effectuer pour économiser de l'énergie et comment les pratiquent-ils ? Nous nous focaliserons alors sur les pratiques et les connaissances énergétiques sur lesquelles les élèves-adolescents peuvent agir, telles que le transport local, le chauffage, le tri sélectif, l'utilisation d'eau chaude ou encore la mise en veille des appareils électroménagers. En considérant le point de vue de l'adolescent lui-

\footnotetext{
${ }^{2}$ Jodelet (1989) présente la représentation comme « une forme de connaissance, socialement élaborée et partagée, ayant une visée pratique et concourant à la construction d'une réalité commune à un ensemble social » (p. 53). Pour Abric (1994), «la représentation fonctionne comme un système d'interprétation de la réalité qui régit les relations des individus à leur environnement physique et social, elle va déterminer leurs comportements ou leurs pratiques. La représentation est un guide pour l'action, elle oriente les actions et les relations sociales » (p. 13).
} 
même sur ses propres pratiques au quotidien, à l'école et à la maison, nous interrogeons également l'origine de ses connaissances et pratiques énergétiques : sont-elles liées à l'enseignement dispensé dans le cadre de leur établissement ou sont-elles liées aux pratiques familiales?

\section{Les représentations et pratiques enseignantes en 2013 : étude 1}

Pour caractériser l'activité et les représentations des enseignants concernant l'éducation à l'énergie, un volet exploratoire a été mené en 2013 à partir d'une étude de cas au sein d'un collège sans action spécifique liée au développement durable. Cinq entretiens semi-directifs ont été réalisés auprès d'enseignants de disciplines différentes en collège (Technologie, SVT, Physique-Chimie, Histoire-Géographie et Documentaliste), en questionnant leurs représentations des économies d'énergie, leur manière de les enseigner, et leur engagement personnel vis-à-vis des économies d'énergie.

Chaque entretien, réalisé dans l'établissement, a été enregistré et retranscrit. Les enseignants interviewés avaient entre 12 et 20 ans d'expérience, sauf l'enseignante de Technologie qui était en fin de carrière. Un verbatim des entretiens a été établi. Puis, une analyse thématique du discours de type papier-crayon (Bardin, 2013) a permis de caractériser :

- le point de vue et les représentations de chaque enseignant ;

- leur engagement personnel vis-à-vis du développement durable et des économies d'énergie en particulier ;

- le type d'activités que chaque enseignant propose ou privilégie en classe et le mode de sollicitation ou d'engagement des élèves envers ces activités.

Nous recensons dans le tableau 1 la synthèse de l'analyse des réponses de chaque enseignant interrogé, explicitée plus précisément dans les parties suivantes, avec pour objectif de comparer leurs représentations, engagements personnels et activités d'apprentissage déclarées.

\begin{tabular}{|c|c|c|c|c|c|}
\hline & SVT & Phys./Chimie & Histoire/Géo. & Techno & Documentaliste \\
\hline $\begin{array}{l}\text { Représentat- } \\
\text { ions }\end{array}$ & Générales & $\begin{array}{c}\text { Concrètes, } \\
\text { pragmatiques }\end{array}$ & $\begin{array}{l}\text { Générales, } \\
\text { échelle globale }\end{array}$ & Pessimistes & Affirmées, concrètes \\
\hline $\begin{array}{c}\text { Activités } \\
\text { réalisées et } \\
\text { modes de } \\
\text { sollicitations } \\
\text { des élèves }\end{array}$ & $\begin{array}{l}\text { Gestes } \\
\text { imposés : } \\
\text { Ordinateurs, } \\
\text { lumière }\end{array}$ & $\begin{array}{c}\text { Exposés, sondages, } \\
\text { calculs } \\
\text { Gestes imposés }\end{array}$ & $\begin{array}{l}\text { Choix et prise } \\
\text { de décision : } \\
\text { études de cas }\end{array}$ & $\begin{array}{c}\text { Vidéos } \\
\text { Gestes } \\
\text { limités } \\
\text { imposés : } \\
\text { papier }\end{array}$ & $\begin{array}{c}\text { Projet } \\
\text { pluridisciplinaire, } \\
\text { réflexion, esprit } \\
\text { critique }\end{array}$ \\
\hline $\begin{array}{c}\text { Engagement } \\
\text { Personnel }\end{array}$ & $\begin{array}{l}\text { Economies } \\
\text { d'énergie } \\
\text { Habitudes de } \\
\text { consommation }\end{array}$ & $\begin{array}{l}\text { Engagement fort } \\
\text { Economies } \\
\text { d'énergie } \\
\text { Habitudes de } \\
\text { consommation }\end{array}$ & $\begin{array}{l}\text { Habitudes de } \\
\text { consommation }\end{array}$ & $\begin{array}{l}\text { Compost, } \\
\text { eau, } \\
\text { recyclage } \\
\text { Contraintes }\end{array}$ & $\begin{array}{c}\text { Co-voiturage, } \\
\text { transports en } \\
\text { commun, circuits } \\
\text { courts, alimentation, } \\
\text { chauffage }\end{array}$ \\
\hline
\end{tabular}

Tableau 1. Comparaison des représentations, activités pédagogiques et engagements personnels des enseignants interrogés 


\subsection{Les représentations enseignantes}

Les représentations vis-à-vis des économies d'énergie sont différentes chez les enseignants interrogés. Alors que certains enseignants ont des réponses floues, d'autres citent des actions concrètes correspondant aux définitions données par Bonfils (2008), Moussaoui $(2009,2011)$ et Zelem (2010), représentations qui traduisent souvent un engagement personnel et professionnel important sur le sujet (enseignant de PhysiqueChimie et Documentaliste).

Plus précisément, dans cette étude, les enseignants d'Histoire-Géographie et de Sciences de la Vie et de la Terre interrogés restent vagues ( une consommation différente »; « une production différente»; " produire d'autres types d'énergie») et parlent des économies d'énergie en se référant à la planète plus qu'à des actions réalisables à court termes, dans leur propre environnement. L'enseignante de technologie semble quant à elle avoir une représentation pessimiste sur le pouvoir d'agir des individus. Tandis que l'enseignante en Physique-Chimie fait, de façon plus concrète, un lien avec "l'isolation des maisons, les transports, le recyclage ", mais signale également que, de son point de vue, il s'agit de «faire attention à ce qu'on achète, de réfléchir à l'impact de ce qu'on consomme, d'où ça vient, ...».

\subsection{Les pratiques de classe}

D'après l'analyse des discours des enseignants interrogés, leurs pratiques semblent liées aux instructions officielles des programmes de 2008. Selon leur discipline, les enseignants déclarent proposer aux élèves des modalités pédagogiques différentes (exposés, gestes imposés, étude de cas, calcul de consommation...) et liées aux activités inscrites dans les programmes, mais ils signalent tous éprouver des difficultés pour mettre en œuvre des situations d'apprentissage développant des gestes efficaces et un esprit créatif chez les élèves-adolescents.

D'après l'analyse des entretiens, peu de liens ou transferts vers la vie quotidienne semblent réalisés. Les élèves vont alors apprendre des "petits gestes », comme l'a montré l'étude de Jeziorski et Ludwig-Legardez (2011), puisque peu de gestes concrets réfléchis collectivement et réalisés librement sont proposés par les enseignants interrogés. Cela questionne les apprentissages effectifs des élèves dans ces conditions: en quoi les enseignants permettent-ils aux élèves d'entrer dans une attitude réflexive et de donner du sens aux apprentissages effectués ?

Néanmoins, il semble difficile pour les enseignants de mener à bien cet enseignement s'ils cherchent à appliquer précisément les programmes scolaires, qui présentent une approche sociétale de l'énergie, trop générale, imprécise et souvent lacunaire, comme le soulignent Bächtold, Munier, Guedj, Lerouge et Ranquet (2014). Seule la documentaliste, moins contrainte par les programmes, travaille en équipe avec d'autres enseignants et propose des projets pluridisciplinaires permettant la réflexion et le développement de l'esprit critique.

\subsection{Des enseignants éco-citoyens ?}

Plus l'engagement personnel de l'enseignant semble fort, plus celui-ci semble faire des liens avec les activités proposées aux élèves. Cependant, les connaissances et pratiques 
pédagogiques des enseignants sur le thème des économies d'énergie paraissent certes étroitement liées à leur engagement personnel mais également à la discipline qu'ils enseignent.

En effet, les enseignants d'Histoire-Géographie, de Sciences de la Vie et de la Terre et de Technologie pratiquent quelque peu les économies d'énergie chez eux, avec des éco-gestes tels que l'isolation de la maison, la consommation locale privilégiée, le compostage, la mise en veille des appareils électriques ou encore les récupérateurs d'eau mais ne proposent aux élèves ni de mettre en pratique, ni de faire des liens avec leur vie quotidienne. Tandis que l'enseignante en Physique-Chimie, qui montre un engagement fort ( $~ \mathrm{Il}$ fait $15^{\circ} \mathrm{C}$ chez nous, on est quand même très économes au niveau de l'énergie, au niveau de l'eau... je fais du covoiturage, du compostage. Et moi, je ne peux pas manger une conserve, c'est pas possible »), privilégie les exposés et sondages en lien avec le quotidien des élèves. En revanche, elle ne propose pas de réelle mise en pratique de la part des élèves. Des applications sont imposées aux élèves dans la classe comme par exemple, l'utilisation d'un bac pour recycler les piles ou de feuilles de brouillons.

Seule la documentaliste, ayant une représentation concrète du développement durable, moins contrainte par les programmes scolaires et très engagée personnellement, semble se permettre la possibilité d'agir au collège, même si elle reconnaît que « les élèves ont les bonnes réponses, mais qu'ils ne veulent pas faire ». Lange (2011) avance en effet l'idée que les comportements des élèves peuvent se heurter à des obstacles épistémiques et sociaux. Garabuau-Moussaoui (2009) insiste par ailleurs sur la spécificité de l'adolescent qui a tendance à « oublier », à dire qu'il a « la flemme ».

\subsection{Quels obstacles à dépasser pour un changement durable?}

Nous constatons, à travers les entretiens réalisés, que les enseignants peinent à travailler la complexité et la transversalité avec leurs élèves, ce qui rejoint les travaux de LeinningerFrezal (2010) affirmant que les enseignants «sortent difficilement de leur fonctionnement scolaire et disciplinaire. Peu se réclament d'une démarche systémique, transversale et critique, structurée autour de plusieurs niveaux d'échelle» (p. 129). Pourtant, cette démarche est indispensable puisque le jeune est en interaction avec divers éléments dans différents moments et aspects de sa vie (à l'école, mais aussi à la maison, avec ses amis, etc.) et les gestes liés aux économies d'énergie dépendent de l'ensemble de ces contextes et milieux.

Il semble par ailleurs exister un décalage entre les représentations des enseignants, leur engagement personnel et les activités réalisées en classe. Seule la documentaliste, moins contrainte par les programmes scolaires, semble être en cohérence entre ses représentations, son engagement personnel et les activités réalisées avec les élèves. Les objectifs et

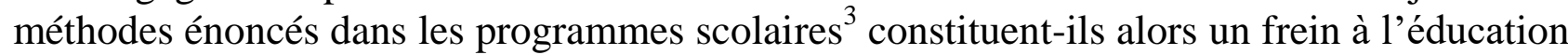
aux économies d'énergie?

La plupart des enseignants du collège étudié se disent en effet contraints par les programmes et évoquent le manque de temps, de compétences et de formation, ce qui

\footnotetext{
${ }^{3}$ cf. travaux Jickling et Wals (2008).
} 
rejoint les travaux de Bächtold et al. (2014) et ceux de Barthes et Alpe (2013). L'Éducation au Développement Durable correspondrait souvent, pour les enseignants, à un projet de mise en norme avec de « bonnes pratiques », de «bons gestes » (Lange, 2012), sans amener les élèves à se questionner. Il serait alors nécessaire de proposer aux enseignants les moyens de faire évoluer leurs représentations vis-à-vis de cet enseignement et de leur donner les moyens d'agir en classe et dans l'établissement, notamment par des actions expérientielles (Lange, 2014).

\section{Connaissances et pratiques des élèves-adolescents en 2014-2015 : étude 2}

Dans un deuxième volet de l'étude, nous avons pris en compte le point de vue des élèves : que retiennent-ils alors des enseignements relatifs aux économies d'énergie ? Les modalités d'enseignement influencent-elles les pratiques des adolescents au quotidien ?

Nous avons interrogé, lors de l'année scolaire 2014-2015, 257 élèves de Troisième (Moyenne d'âge $=13,84 ; \mathrm{ET}=0,84$ ) de la région Centre-Val de Loire (France), dont 48\% de garçons et $49 \%$ de filles ( 9 élèves n'ayant pas répondu à cette question). Parmi les élèves interrogés (cf. tableau 2), malgré de nombreuses non-réponses (103 élèves n'ont pas répondu à la question interrogeant leur lieu d'habitation), nous notons un équilibre entre ceux qui habitent en milieu urbain $(32,3 \%)$ et ceux qui demeurent en milieu rural $(27,6 \%)$. Cet équilibre se retrouve par ailleurs dans le choix des établissements, puisque parmi les cinq établissements scolaires dans lesquels sont inscrits les élèves interrogés, deux sont situés en centre-ville, deux en zone péri-urbaine et un en milieu rural. En revanche, seulement $26,8 \%$ des élèves de l'échantillon vivent en appartement contre 64,6\% qui résident en maison (22 non-réponses).

\begin{tabular}{|c|c|c|c|c|}
\hline $\begin{array}{c}\text { Lieu d'habitation } \\
\text { des élèves }\end{array}$ & $\begin{array}{c}\text { Établissement } \\
\text { Centre-ville }\end{array}$ & $\begin{array}{c}\text { Établissement } \\
\text { Péri-urbain }\end{array}$ & $\begin{array}{c}\text { Etablissement } \\
\text { Rural }\end{array}$ & Total \\
\hline Urbain & 51 & 29 & 3 & $\begin{array}{c}83 \\
(32,3 \%)\end{array}$ \\
\hline Rural & 29 & 19 & 23 & $\begin{array}{c}71 \\
(27,6 \%)\end{array}$ \\
\hline Appartement & 27 & 41 & 1 & $\begin{array}{c}69 \\
(26,8 \%)\end{array}$ \\
\hline Maison & 84 & 52 & 30 & $\begin{array}{c}166 \\
(64,6 \%)\end{array}$ \\
\hline Total élèves & 127 & $\begin{array}{c}97 \\
(49,4 \%)\end{array}$ & $33 \%)$ & 257 \\
\hline
\end{tabular}

Tableau 2. Lieu d'habitation des élèves en fonction de l'établissement dans lequel ils sont inscrits (fréquences d'effectifs et pourcentages cumulés)

Afin de répondre aux objectifs de l'étude, un questionnaire a été élaboré. Il comprend une échelle de type Likert qui interroge les adolescents quant à leur pratique ${ }^{4}$ des éco-gestes ; une échelle évaluant leurs connaissances dans ce domaine; des questions ouvertes sur la

\footnotetext{
${ }^{4}$ Les éco-gestes ne sont alors pas mesurés directement mais uniquement via la déclaration des élèves, ce qui nous permet d'appréhender davantage leurs représentations et le sens qu'ils leur accordent, plus que leur pratique réelle.
} 
pratique du jeune dans et hors l'école, sur les pratiques familiales et enfin des questions d'ordre socio-biographique (genre, âge, lieu d'habitation, profession des parents).

La première échelle validée pour cette étude (coefficient de cohérence interne Alpha de Cronbach $=0,67$ ) comporte 14 items correspondant aux principaux gestes quotidiens que peuvent pratiquer les élèves, allant de la consommation d'eau, de chauffage, d'électricité à la maison aux moyens de transports utilisés par le jeune (intitulés «éco-gestes individuels »). Des gestes davantage liés aux pratiques familiales (intitulés «éco-gestes Maison ») font également partie des items proposés dans l'échelle (À la maison, on récupère l'eau de pluie ; On mange des produits locaux et/ou du jardin). Trois items correspondent à des gestes qui ne permettent pas d'économiser de l'énergie (Je reste longtemps sous la douche; Je laisse toujours branchés les chargeurs de téléphone, d'ordinateur...; J'aime mettre mon chauffage bien fort). Ces items ont alors été inversés lors du codage (Jamais correspondant alors à 4 points, tandis que la réponse Toujours vaut 1 point). Le calcul des points pour chaque item permet d'obtenir un score global de gestes d'économie d'énergie signalés comme étant pratiqués par l'adolescent (intitulés « éco-gestes globaux »).

Par ailleurs, afin de mesurer les connaissances que les jeunes ont sur les éco-gestes, une échelle également composée de 14 items a été élaborée et validée (coefficient de cohérence interne Alpha de Cronbach $=0,84$ ). Les élèves doivent dire si le geste proposé permet à leur avis d'économiser de l'énergie. Les items sont les mêmes que pour l'échelle précédente (14 gestes proposés; question : à ton avis, ce geste permet-il d'économiser de l'énergie ? Le jeune peut répondre oui, non ou je ne sais pas). Un point est attribué à chaque bonne réponse. Un score global est alors obtenu: plus le score est élevé, plus l'élève a une connaissance de ce qui permet l'économie d'énergie.

Afin de comparer les moyennes des scores obtenus pour chacune de ces échelles en fonction des variables de notre étude, telles que le genre, les milieux de vie scolaire et familiale, une analyse statistique de comparaison de moyennes (ANOVA) à l'aide du logiciel SPSS.14 a été effectuée.

Nous présentons ici les résultats se centrant sur les pratiques des adolescents au quotidien, puis au collège plus particulièrement et, enfin sur leurs connaissances relatives aux moyens d'économiser de l'énergie.

\subsection{Pratiques des adolescents au quotidien}

De façon générale (cf. tableau 3), les gestes liés aux économies d'électricité semblent être les plus pratiqués par les collégiens interrogés (score moyen $=3 ; \mathrm{ET}=0,6$ ). Cela peut s'expliquer par le fait que de nos jours, les jeunes sont de plus en plus en contact avec les appareils électriques et de mieux en mieux formés et informés sur leur utilisation.

Tandis que les gestes liés à une pratique familiale (éco-gestes maison) semblent être les moins pratiqués (score moyen $=2,45 ; \mathrm{ET}=0,7$ ). On peut imaginer que les adolescents se sentent moins concernés par ces gestes (Garabuau-Moussaoui, 2009), car ils sont imposés ou mis en œuvre par la famille. 


\begin{tabular}{|c|c|c|c|c|}
\hline Gestes & Score moyen & Écart-Type & Min & Max \\
\hline Électricité & 3 & 0,6 & 1 & 4 \\
\hline Chauffage & 2,59 & 0,9 & 1 & 4 \\
\hline Eau & 2,75 & 0,6 & 1 & 4 \\
\hline Éco-gestes globaux $^{\mathbf{5}}$ & 2,75 & 0,4 & 1,29 & 3,93 \\
\hline Éco-gestes « Individuels » $^{\mathbf{6}}$ & 2,87 & 0,4 & 1,4 & 4 \\
\hline${\text { Éco-gestes « Maison }{ }^{7}}^{\text {Éconnaissances }}$ & 2,45 & 0,7 & 1 & 4 \\
\hline
\end{tabular}

Tableau 3. Moyenne des scores aux différents items de l'échelle «pratique des éco-gestes » catégorisés en fonction du type de consommation d'énergie et moyenne des scores de l'échelle « connaissances des éco-gestes ».

Néanmoins, l'échelle proposée aux élèves amène à nuancer nos propos : elle a pu orienter les réponses des collégiens, les gestes étant proposés par les chercheurs. Également, certains gestes présents dans l'échelle peuvent davantage être mis en œuvre dans un lieu de vie qui s'y prête, tel qu'une maison pour la gestion des économies d'eau et d'électricité ou encore la campagne pour l'utilisation d'un jardin par exemple.

Nous n'observons aucun lien significatif entre les différentes dimensions de pratique des éco-gestes et le genre ainsi que l'âge des adolescents. La pratique des éco-gestes est alors corrélée à d'autres facteurs davantage liés au milieu de vie, de façon significative ( $\mathrm{p}<0,001)$. Les élèves ayant un parent agriculteur ou cadre, vivant dans une maison et à la campagne pratiquent plus souvent des éco-gestes que les élèves habitant en appartement et en ville $(\mathrm{p}<0,001)$.

\subsection{Pratiques des éco-gestes au collège}

Seulement $9,3 \%$ des élèves interrogés signalent réaliser des gestes liés aux économies d'énergie dans leur établissement scolaire. Ces gestes déclarés concernent principalement le fait d'éteindre les ordinateurs, les lumières et de mettre les papiers à la poubelle. Si le pourcentage est peu élevé et nous questionne quant à la mise en pratique proposée par les professionnels de l'éducation, cela montre que certains élèves semblent tout de même être encouragés à développer des habitudes, ce qui est attendu pour réaliser au quotidien des écogestes. Mais, ces gestes pourraient être questionnés : encouragent-ils une responsabilité et un engagement citoyen ? En effet, nous pouvons nous demander si les jeunes leur donnent du sens ou s'ils les mettent en œuvre par simple habitude.

De plus, nous repérons un lien significatif entre la pratique des adolescents dans leur quotidien et leur pratique en milieu scolaire (cf. tableau 4) : les élèves affirmant réaliser des

\footnotetext{
${ }^{5}$ Correspondant à l'ensemble des éco-gestes proposés dans l'échelle (score global).

${ }^{6}$ Correspondant à l'ensemble des éco-gestes pouvant être pratiqués par l'adolescent seul (exemple : «J'utilise la lumière du soleil pour travailler dans la journée »).

${ }^{7}$ Correspondant à l'ensemble des éco-gestes pratiqués au sein de la famille (exemple : «On trie les déchets à la maison $\gg$ ). 
éco-gestes à l'école ont un score moyen de pratique des éco-gestes globaux significativement $(\mathrm{p}<0,001)$ plus élevé $(\mathrm{M}=2,9)$ que les élèves ne pensant pas réaliser d'éco-gestes à l'école $(\mathrm{M}=2,5)$.

\begin{tabular}{|c|c|c|c|c|c|c|c|}
\hline & \multicolumn{4}{|c|}{ Pratique scolaire } & \multirow{3}{*}{ ddl } & \multirow{3}{*}{$\mathbf{F}$} & \multirow{3}{*}{$p$. } \\
\hline & \multicolumn{2}{|c|}{ Oui } & \multicolumn{2}{|c|}{ Non } & & & \\
\hline & $\mathbf{M}$ & E.T. & $\mathbf{M}$ & E.T. & & & \\
\hline Éco-gestes globaux & 2,9 & 0,4 & 2,5 & 0,4 & $4 ; 252$ & 5,3 & 0,001 \\
\hline $\begin{array}{l}\text { Éco-gestes } \\
\text { individuels }\end{array}$ & 3,1 & 0,4 & 2,7 & 0,4 & $4 ; 252$ & 4,5 & 0,001 \\
\hline Éco-Gestes maison & 2,6 & 0,7 & 2,2 & 0,7 & $4 ; 252$ & 2,2 & 0,06 \\
\hline
\end{tabular}

Tableau 4. Comparaison de moyennes des scores de chaque dimension des éco-gestes quotidiens en fonction de la pratique de l'élève à l'école.

Tout comme la pratique en famille, la mise en pratique au sein de l'établissement scolaire semble donc nécessaire pour encourager les jeunes à développer des éco-gestes, ainsi que l'exemplarité des professionnels de la communauté éducative ou encore la communication avec les familles pour permettre aux élèves de faire des liens entre leurs différents milieux de vie.

\subsection{Connaissances des adolescents sur les éco-gestes}

Concernant les connaissances des gestes liés aux économies d'énergie, nous notons de façon générale un score supérieur à la moyenne (score moyen pour l'ensemble de l'échantillon $=8,28 / 14$ ), ce qui signifie que les élèves interrogés connaissent globalement les éco-gestes, leurs effets et sont capables de les mettre en œuvre. Cela rejoint les travaux de Kovacs (2012) ayant réalisé des entretiens avec des collégiens : «les élèves évoquent volontairement les gestes et les connaissances acquis dès le primaire : le tri des déchets, l'importance des transports en commun et le vélo, les économies d'énergie et d'eau à la maison » (p.78). Cette connaissance ne semble toutefois que partielle, ce qui questionne sur la façon dont les élèves comprennent les enjeux des économies d'énergie. Nous sommes alors amenés, à nouveau, à nous demander si les enseignements liés aux économies d'énergie permettent bien aux élèves d'appréhender le sens et la finalité des éco-gestes.

Enfin, nous constatons un lien significatif entre les connaissances des élèves sur les écogestes et leur pratique (individuelle et collective) : plus les élèves pratiquent dans différents milieux de vie (école, maison, ...), plus ils ont une connaissance exacte des éco-gestes $(\mathrm{p}<0,001)$. Il semble alors important d'éduquer à la fois à la connaissance et à la pratique, ce qui manque peut-être en milieu scolaire.

\subsection{Du point de vue des collégiens aux évolutions possibles?}

Nos résultats mettent en avant l'idée d'une interdépendance entre les pratiques des adolescents dans différents lieux de vie et leurs connaissances. Il semble alors important d'éduquer les élèves à la connaissance des économies d'énergie mais aussi de développer 
l'autonomie des adolescents à la pratique d'éco-gestes, notamment en les impliquant, en leur permettant de donner du sens à ces actes et en encourageant cette pratique dans leurs différents milieux de vie. Il parait également important d'amener les professionnels de l'éducation à faire preuve d'exemplarité. En effet, la citoyenneté ne se construit pas dans le discours ou les connaissances, mais dans les actes et dans l'articulation entre savoirs et pratiques (Audigier, 2007).

Or, les activités concrètes permettant l'engagement des élèves et l'appropriation des écogestes avec des transferts vers la vie quotidienne, donc applicables en fonction du contexte, sont peu proposées par les enseignants. Pourtant, la participation active à des pratiques environnementales est un facteur de motivation important et permet le transfert vers des pratiques dans d'autres contextes (Gruslin, 2007). Il apparait donc indispensable d'outiller les élèves pour impulser un changement réel et durable de comportement (Bartiaux, 2009) et ainsi les inscrire davantage dans une "société de modération » (Moussaoui, 2007). Il sera néanmoins essentiel de questionner les pratiques enseignantes, notamment dans le cadre de la formation initiale et continue, pour éviter de développer chez les élèves le sentiment de «résignation à une forme d'engagement comportemental quelque peu automatisé (...) mettant l'accent sur la responsabilité individuelle » (Kovacs, 2012, p. 78).

\section{Education à l'énergie au collège: qu'est-ce qui pourrait permettre le changement?}

Dans la présente étude, les discours des enseignants interrogés en 2013 sont à mettre en lien avec les recommandations des programmes scolaires de 2008, alors en vigueur. En effet, ces programmes, centrés principalement sur les connaissances, mettaient peu en avant des éléments ou activités consacrés aux éco-gestes. Le lien avec l'EDD était également difficile à repérer. Nous pouvons alors comprendre le point de vue des enseignants interrogés dans cette étude lorsqu'ils signalent proposer peu de mises en situation ou de projets puisqu'ils s'attachent à suivre les programmes scolaires. L'approche utilitariste, liée à une certaine vision du monde, et «morcelée » qui peut en découler a alors été questionnée par de nombreux chercheurs dans le domaine des questions socialement vives (cf. Barthes et Jeziorski, 2012). Une éducation critique et transversale serait essentielle pour répondre aux questions socialement vives, en interrogeant notamment l'organisation collective de la société. Nous pouvons supposer que ce point de vue soit effectif avec l'entrée en vigueur en septembre 2016 des nouveaux programmes scolaires (2015), le nouveau socle commun des connaissances et des compétences (SCCC) ayant entrainé une évolution des attentes vis-àvis des apprentissages. L'application de ces nouveaux programmes scolaires irait ainsi dans le sens de la transition énergétique puisque ces derniers devraient permettre de faire évoluer les représentations des élèves et les pratiques des enseignants relativement aux économies d'énergie, à l'éco-citoyenneté et plus généralement au développement durable ${ }^{8}$.

Aussi, cette étude pourrait être prolongée de façon longitudinale auprès des élèves pour caractériser à moyen terme l'influence d'un enseignement à l'EDD, en constante évolution,

\footnotetext{
${ }^{8}$ Les nouveaux programmes (2015) insistent en effet davantage sur les liens avec la vie quotidienne et la formation du futur citoyen. Présentant une véritable progression entre le cycle 3 et le cycle 4, ils cherchent de surcroît à croiser différentes disciplines autour d'un même objectif. Ils encouragent par ailleurs un travail sur la posture, l'attitude, l'ouverture au monde et le développement de l'esprit critique chez les élèves.
} 
sur les représentations sociales et comportements des jeunes au quotidien. Il s'agirait alors de repérer, à travers une enquête qualitative, si les jeunes adoptent une posture réflexive visà-vis des économies d'énergie ou s'ils maintiennent une simple pratique des « bons gestes ». Il serait également nécessaire d'analyser de plus près les pratiques pédagogiques des enseignants au regard de la transition énergétique, en prenant en compte le contexte socioculturel et politique dans lequel ils vivent et travaillent. Dans une prochaine étude, nous envisageons d'étendre ce premier travail exploratoire en questionnant de façon quantitative l'ensemble des enseignants de collège d'une même académie sur leurs représentations vis-àvis des économies d'énergie et leurs pratiques pédagogiques afin de repérer notamment des liens entre représentations sociales, engagement et prise en compte des pratiques pédagogiques nécessaires à l'éducation à la citoyenneté.

Notre étude nous amène par ailleurs à interroger la co-construction des actions éducatives entre une diversité d'acteurs (famille, école, partenaires extérieurs). En effet, la pratique des éco-gestes semble liée au milieu de vie sociale, aux pratiques familiale et scolaire. Il paraît alors nécessaire d'appliquer le principe de coéducation entre l'école et la famille. Les changements de comportement attendus des jeunes générations ne peuvent se développer que si l'école, la société et la famille ont des discours, des pratiques et des valeurs communs, cohérents (Audigier, 2007). Le travail en équipe, mené par les enseignants, induit et favorisé par l'interdisciplinarité, mais aussi les liens avec la vie quotidienne et les transferts vers celle-ci devraient être moteurs pour ce(s) changement(s) et pourraient être questionnés dans une prochaine recherche.

En outre, nos résultats nous amènent à penser la nécessité de développer à la fois certaines habitudes et connaissances importantes pour la mise en œuvre d'un changement durable, mais de travailler aussi de façon plus globale à l'autonomisation des jeunes en éduquant à la complexité. La démarche de projet (Lange, 2014) pourrait notamment encourager la transdisciplinarité et une forme d'autonomisation et de capacité d'agir chez l'élève, gage d'une éco-citoyenneté mise en acte. En vue de permettre les changements de comportement des élèves, les enseignants vont en effet être amenés à développer chez ces derniers leur esprit critique à travers des questionnements/réflexions (capacité à faire des choix, s'engager, ...) et à encourager à la fois leur créativité et la pratique au sein même de l'établissement scolaire. La formation des enseignants devra alors selon nous, encourager chez ces derniers une attitude réflexive vis-à-vis de la relation entretenue avec le monde et la citoyenneté, en s'appuyant notamment sur les représentations sociales des étudiants et en éclairant le caractère complexe des questions socialement vives.

Enfin, considérer l'élève comme «auteur», c'est-à-dire lui permettre de se sentir responsable de ses choix, de ses décisions, de ses actes en le faisant pleinement participer (Lange et Janner-Raimondi, 2015), pourrait être un levier pour encourager l'autonomie des élèves-citoyens au sein d'un établissement scolaire engagé. En effet, l'engagement des adolescents dépend des contraintes imposées mais également du sens donné aux actes, ce qui peut être travaillé en milieu scolaire. Les représentations des adolescents-élèves sur leur pouvoir d'agir peuvent évoluer, notamment par une prise de conscience environnementale et un engagement réel citoyen. Car, comme le dit Audigier (2007), c'est « en citoyennant que l'on devient citoyen ». 


\section{Bibliographie}

ABRIC, J.-C. (1994, Dir.). Pratiques sociales et Représentation. Paris : PUF.

AUDIGIER, F. (2007). L'éducation à la citoyenneté dans ses contradictions. Revue internationale d'éducation de Sèvres, $n^{\circ} 44,25-34$.

BARdin, L. (2013). L'analyse de contenu, Paris : PUF, Paris.

BARTHES, A., JEZIORSKI, A. (2012). What kind of critical university education for sustainable development? A comparative study of European students and social representations. Journal of Social Science of Education: Volume 11, Number 4.

BARThes, A., AlPe, Y. (2013). Le curriculum caché du développement durable. Dans J-M. Lange (dir.), Actes du Colloque international «Education au développement durable : appuis et obstacles à sa généralisation hors et dans l'Ecole », Rouen, 26, 27 \& 28 novembre 2012, Penser l'éducation, Hors-série, 485-502.

BARTIAUX, F. (2009). Changer les comportements individuels liés à l'énergie: problématisations et instruments politiques. Bruxelles : Défi énergie.

Bächtold, M., Munier, V., Guedj, M., Lerouge, A. et RAnquet, A. (2014). Quelle progression dans l'enseignement de l'énergie de l'école au lycée ? Une analyse des programmes et des manuels. Recherches en Didactique des Sciences et Technologies (RDST), $n^{\circ} 10,63-92$.

BonfILs, S. (2008). Stratégies énergétiques pour le développement durable. Liaison énergie francophonie, $78,34-40$.

GARABUAU-MousSAOUI, I. (2009). Vers une génération de la modération ? Pratiques, représentations et systèmes de consommation énergétique selon les âges sociaux. Dans M. Dobré et J. Salvador (dir.), Consommer autrement la réforme écologie des modes de vie (pp. 253-262). Paris : L'Harmattan.

GARABUAU-MoussaOUI, I. (2011). L'énergie est-elle un enjeu de pouvoir dans la famille ? Dans S. Barrey et E. Kessous (dir.), Consommer et protéger l'environnement Opposition ou convergence (pp. 67-89). Paris : L'harmattan, Paris.

Garabuau-Moussaoui, I., BartiauX, F. et Filiastre, M. (2009). Entre école, famille et médias, les enfants sont-ils des acteurs de transmission d'une attention environnementale et énergétique ? Une enquête en France et en Belgique. Dans N. Nurnay et A. Llein (dir.), Figures contemporaines de la transmission (pp. 105-120). Namur : Presses Universitaires de Namur.

GRUSLIN, M. (2007). Vivre des projets environnementaux en formation initiale, pour stimuler la pratique en éducation relative à l'environnement, Éducation relative à l'environnement, $n^{\circ} 6,237-243$.

JEZIORSKI, A., LUDWIG-LEGARDEZ, A. (2011). Éducation au développement durable : la difficulté de concevoir une action éducative interdisciplinaire. Colloque international francophone "Le développement durable : débats et controverses », Université Blaise Pascal, Clermont-Ferrand, 15 et 16 décembre 2011.

JiCKLING, B., WALS, A.-E.-J. (2008). Globalization and environmental education: looking beyond sustainable development. Journal of curriculum studies, 1 (40), 1-21.

JODELET, D. (1989, Ed.). Les représentations sociales. Paris : PUF.

Kovacs, S. (2012). Engager et enrôler les jeunes dans la lutte contre le changement climatique : le documentaire jeunesse et l'attitude des collégiens d'aujourd'hui. Communication \& langages, $n^{\circ} 172$, 69 81.

LANGE, J.-M. (2008). L'éducation au développement durable au regard des spécialités enseignantes. Aster, $n^{\circ} 46,123-154$.

LANGE, J.-M. (2011). Éducation au développement durable : éléments pour une problématisation de la formation des enseignants. Carrefours de l'éducation, $n^{\circ} 1 \mathrm{HS}, 71-85$. 
LANGE, J.-M. (2012). Pour un curriculum de l'éducation au développement durable : entre actions de participations et démarches multi-référentielles d'investigation d'enjeux. Rapport aux savoirs, éducation relative à l'environnement et au développement durable. Éducation Relative à l'Environnement : Regards-Recherche-Réflexions, $n^{\circ} 11,41-60$.

LANGE, J.-M. (2014). Des dispositions des personnes aux compétences favorables à un développement durable : place et rôle de l'éducation. Dans A. Diemer et C. Marquat (dir.), Éducation au développement durable. Enjeux et controverse (pp. 163-182), Louvain-la-Neuve : De Boeck.

LANGE, J.-M., JANNER-RAIMONDI, M. (2015). Education à un développement durable et principe de responsabilité : enjeux éducatifs et démocratiques. Colloque International Francophone "Former au monde de demain », Clermont-Ferrand, 2-3 avril 2015.

LEGARDEZ, A. (2004). L'utilisation de l'analyse des représentations sociales dans une perspective didactique : l'exemple de questions économiques. Revue des sciences de l'éducation, $n^{\circ} 30$ (3), 647-665.

LEINNINGER-FREZAL, C. (2010). Éducation à l'environnement vers le développement durable : enseignant en difficulté. Dans M.-C. Zélem, O. Blanchard, et D. Lecomte (dir.), L'éducation au développement durable de l'école au campus (pp. 123-129). Paris : L'Harmattan.

LOI ${ }^{\circ}$ 2015-992 du 17 août 2015 relative à la transition énergétique pour la croissance verte, parue au JORF $\mathrm{n}^{\circ} 0189$ du 18 août 2015.

Moussaoui, I. (2007). De la société de consommation à la société de modération : ce que les Français disent, pensent et font en matière de maîtrise de l'énergie. Les Annales de la recherche urbaine, $n^{\circ} 103$, 112-119.

SECA, J.-M. (2010). Les représentations sociales. Paris : Armand Colin.

ZELEM, M.-C. (2010). Politiques de maîtrise de la demande d'énergie et résistances au changement. Une approche socio-anthropologique. Paris : L'Harmattan. 